\title{
Machine Learning Tools for Engineering Processes Modelling
}

Received: December 26, 2016; Accepted: December 27,2016; Published: December 31, 2016

\section{Introduction}

In some cases, modelling engineering systems (and not only) using classical techniques (statistical and phenomenological approaches) is a difficult task, especially when taking into consideration their complexity and the multiple interactions that take place internally. In this context, alternative solutions that can efficiently and easily solve these systems are necessary. The area of Machine Learning (belonging to the Artificial Intelligence domain) can provide such techniques, the best known examples being represented by Artificial Neural Networks and Support Vector Machines. Various studies proved their efficiency in many cases but it must be taken into consideration that these approaches are not panacea and their use must be carefully evaluated.

Models of specific systems have various applications that range from providing the base for optimization to generating predictions that can be useful for control and decision making. Unfortunately, not all the systems can be easily modelled and solved. In the engineering area, the main reasons for this difficulty are related to: i) Complexity; ii) Incomplete knowledge related to the phenomenology (chemical and physical interactions that take place in the systems); iii) High order derivatives. These problems are especially related to the phenomenological models (deterministic), literature presenting other two types of models: i) Statistical-stochastic (based on the principle of uncertainty); and ii) Empirical models (directly related to the functional relationship between variables and parameters through empirical data) [1].

The majority of modelling and classification tools from Machine Learning area (Artificial Neural Networks -ANNs-, Support Vector Machines -SVM-, Decision Trees Learning -DT, k-nearest neighbours -k-NN- and so on...) belong to the group of empirical models and do not suffer from the drawbacks of the other approaches. They also have a good performance and with little modifications can be applied to systems with various characteristics. In this context, they come as good alternatives, especially when the other approaches fail to provide acceptable results.

ANN's are inspired from the biological brain and they rely on the inner structure of available data sets [2]. SVMs have their roots in statistical learning and optimization methods [3] and they are usually used for classification purposes although they also have regression capabilities. DT are acyclic-directed graphical

\section{Elena Niculina Dragoi}

Department of Chemical Engineering, Applied Informatics and Automation Group, Gheorghe Asachi Technical University of Iasi, Romania

\author{
Corresponding author: \\ Elena Niculina Dragoi
}

elenan.dragoi@gmail.com

Assistant Professor, Faculty of Chemical Engineering and Environmental Protection, Department of Chemical Engineering, Applied Informatics and Automation group, Gheorghe Asachi Technical University of lasi, Romania.

Tel: 0232278683

Citation: Dragoi EN. Machine Learning Tools for Engineering Processes Modelling. Int J Appl Sci Res Rev. 2016, 3:6.

models that based on symbolic representation solve classification problems [4] k-NN is a simple algorithm, a type of instance based learning.

ANN's do not significantly differ from a number of statistical models, the learning and training phases being similar to the parameter estimation phase in conventional statistical models [5]. Distinctively from statistical models (that generally have too restrictive rules) ANN's are flexible, can have a complex structure (and consequently be more representative) and can be highly nonlinear (being able to model any type of function) [6]. Also, ANN's have a direct manner to acquire knowledge about the system (through learning), can work with continuous data, can be regarded as 'black-boxes', the solutions can be robust and have a high degree of accuracy [7]. Due to these characteristics it was widely applied to model different types of systems, literature presenting various examples and reviews related to the use of ANN's in engineering [6,8-12]. However, it must be taken into consideration that ANN's have a series of drawbacks and their easiness of use can be misleading. Consequently, it is recommended that a careful analysis of the system must be performed previous to the application of the modelling procedure. The main drawbacks of the ANN's consists: i) The training data should (ideally) cover the entire domain (range) of the system; ii) 
The design (topology and internal parameter determination) in based on very few precise rules (especially topology); iii) There is no guarantee of finding a near optimal solution; and iv) The solution provided is difficult to rationalize [7].

SVM, a supervised learning method, aims to find a classification hyperplane that meets the requirements [13]. The three main essential elements of SVM that ensure its success (by overcoming problems such as curse of dimensionality and over-fitting) are: Principle of maximal margin, dual theory and kernel trick [3]. Although they have good theoretical foundations and have a series of advantages that cannot be overlooked, SVM's are difficult to implement and their parameters are not easy to find, still existing no consensus and contradictory opinions related to this aspect [14].

Among all the approaches from Machine Learning that can be used for modelling, ANN's and SVM are the most known and widely used. This does not imply that the other methods are not as fast or efficient, it is just that they are older approaches (ANN proposed in 1943 and SVM in the late 1960) and they were implemented in various software that made their use simpler and easier. The main difference between the two is represented by the risk minimization principle used (ANN implements the empirical risk minimization and SVM the structural risk minimization) [14]. Consequently, the performance of each approach on the same system is different, depending on a variety of factors such as dimensionality, type of objective, manner in which the performance is measured, and so on. As a result, attention must be paid to all these aspects in order to ensure that the solution provided by the selected methodology is acceptable. Their advantages make them sough after approaches but, in order to not get trapped into the No Free Lunch theorem (that states that if an algorithm performs well on a specific type of problem that does not necessarily means that it will perform well on another type), one must also take into consideration their problems (disadvantages) and suitability to the system being modelled. 


\section{References}

1 Buso A, Giomo M (2011) Mathematical modeling in chemical engineering: a tool to analyse complex systems. INTECH Open Access Publisher.

2 Mi XC (2005) Testing the generalization of artificial neural networks with cross-validation and independent-validation in modelling rice tillering dynamics. Ecol Model 181: 493-508.

3 Tian YJ, Shi Y, Liu XH (2012) Recent advances on support vector machines research. Technol Econ Dev Econ 18: 5-33.

4 Sok HK (2016) Multivariate alternating decision trees. Pattern Recogn 50: 195-209.

5 Das SK, Biswal RK, Sivakugan N, Das B (2011) Classification of slopes and prediction of factor of safety using differential evolution neural networks. Environ Earth Sci 64: 201-210.

6 Himmelblau D (2000) Applications of artificial neural networks in chemical engineering. Korean J Chem Eng 17: 373-392.

7 Yardimci A (2009) Soft computing in medicine. Appl Soft Comput 9: 1029-1043.
8 Dragoi EN, Curteanu S, Lisa C (2012) A neuro-evolutive technique applied for predicting the liquid crystalline property of some organic compounds. Eng Optim 44: 1261-1277.

9 Dragoi EN, Curteanu S, Fissore D (2013) On the Use of Artificial Neural Networks to Monitor a Pharmaceutical Freeze-Drying Process. Dry Technol 31: 72-81.

10 Curteanu S, Suditu GD, Buburuzan AM, Dragoi EN (2014) Neural networks and differential evolution algorithm applied for modelling the depollution process of some gaseous streams. Environ Sci Pollut Res 21: 12856-12867.

11 Marini F (2008) Artificial neural networks in chemometrics: History, examples and perspectives. Microchem J 88: 178-185.

12 Vinicius GM, Kathia MH, Alberico BFDS (2013) Applications of Artificial Neural Networks in Chemical Problems.

13 Ding S, Zhu Z, Zhang X (2015) An overview on semi-supervised support vector machine. Neural Comput Appl, pp: 1-10.

14 Lahiri SK, Ghanta KC (2008) The support vector regression with the parameter tuning assisted by a differential evolution technique: study of the critical velocity of a slurry flow in a pipeline. Chem Ind Chem Eng Quart 14: 191-203. 